\title{
Assisting an Elderly with Early Dementia Using Wireless Sensors Data in Smarter Safer Home
}

\author{
Qing Zhang ${ }^{1}$, Ying $\mathrm{Su}^{2}$, and Ping $\mathrm{Yu}^{3}$ \\ ${ }^{1}$ Australian e-Health Research Centre/CSIRO Computational Informatics, Australia \\ ${ }^{2}$ Institute of Scientific and Technical Information of China, China, 100038, Beijing, China \\ ${ }^{3}$ Health Informatics Research Laboratory, University of Wollongong, Australia \\ qing.zhang@csiro.au, suy.rspc@istic.ac.cn, ping@uow.edu.au
}

\begin{abstract}
The primary aim of this study is to develop a Smart Assistive Living (SAL) platform to enable old people suffering from dementia to stay at home as long as possible. The technology used includes wireless sensor network and broadband network connectivity. The assistive technology aggregates sensor information about environmental, cognitive, physical and physiological factors and integrate them to support the delivery of telehealth and telecare services to older people with mild dementia. The plan for the testing and implementation of the technology and commercial roll out is also briefly outlined.
\end{abstract}

Keywords: Wireless Sensor Network, Smart home, Activity recognition, Activity of daily living, ADL, Aged carer, Community care, Time series analysis, Information quality.

\section{Introduction}

The looming dementia epidemic has plagued all nations around the world, with its social and economic impact widely acknowledged by World Health Organisation, health organizations and governments. For example, about 14 million people in the Asia Pacific Region, the largest continent with more than half of the world population, are plagued by dementia. Alzheimer's Disease International (ADL) estimated that by 2050 the amount of people with dementia will be tripled to 65 millions.

The cost of dementia in 2003 was $\$ 60.4$ billion for an estimated 12.6 million people with dementia in Asia Pacific region. With an estimated cost of around $1 \%$ of GDP, dementia will become the third largest source of health and residential aged care spending in Australia within two decades. The devastating impact of dementia on the social and public health systems is not only due to it being the most disabling of all chronic diseases, but also its enormous economic impact on health system, care services, and productivity losses. Therefore, dementia presents significant challenges to policymakers and public healthcare system.

While there is no cure to dementia, efforts can be made to delay or reduce the number of people suffering from it. The possible preventive and interventive 
mechanisms include improving social and physical living environment to prevent or slow down the progression of the disease, early interventions (lowering incidence), and improvements in treatment and care to increase survival.

Wellness is about well-being of people. It is indicated by the capability of people in performing their daily activities of living and work. A wellness determination process helps Data related to the wellness indices and behavior recognition can guide the healthcare professionals to identify the variations of people's performance on maintaining or improving health and wellness.

To address the challenge taking the advantage of information and communication technology, we propose a solution called "Smarter and Safer Home" that we hope to enable old people with early dementia (PWED) to remain independently in their homes as long as possible. The second aim of our solution is to enhance quality of life (QoL) for these people, as well as QoL for their family carers. Our strategy is to deploy environmental sensors in their homes, acting as non-intrusive monitoring devices for human behaviour. These sensors are expected to continuously stream data to our central server, where we can then extract and analyse these data to identify the pattern of a person's daily activities. Through the mechanism of machine learning, new activities will be benchmarked against the established patterns to identify those 'abnormal' situations. Once an abnormal signal was detected, an alert will be provided to family carers, such as unattended stove or bathroom flooding. We hope that the 'signal' from the sensors will notify, motivate, and encourage older people and their family carers to take appropriate precaution or intervention. Our proposed technology will lead to new method in assessing the severity of dementia, early detection of abnormal health conditions and development of targeted prevention or early intervention. This will again lead to the reduction of occurrence of behavioural or psychological symptoms and relief burden for family carers to look after their beloved ones. The ultimate goal is to maintain the ADL of people with dementia, improve their QoL and QoL for their family members. This paper will outline the design of our smart home and the plan to implement it.

\section{The Architecture of the System}

We employ the Smarter Safer Home system developed at CSIRO [4] to collect and analyse sensor data from homes of dementia patients. The system consists of two components: (i) Wireless Sensor Network (WSN) for data transmission and (ii) intelligent home monitoring system to collect sensor data, perform data analysis and present results to end user.

\subsection{Wireless Sensor Network}

Although there are many previous work on monitoring ADL of people with dementia through wearable sensors such as RFID markers and wearable accelerometers detecting falls $([1,2,3])$, the innovative aspect of our proposed solution is to place the sensors in the person's living environment, instead attached to their body for 
non-intrusive monitoring of human behavior. The two advantages of this solution are: First, wearable sensors are not always convenient for users to carry around. This is particularly relevant for people with mild dementia as they easily forget things; second, sensors placed in environment is out of the sight of the users, thus has less interference with their daily lives. Third, the data recorded by sensors are anonymous, containing very little information about personal privacy. Table 1 describes all types of in-house sensors that will be used in our smart home system.

Table 1. List of environmentally placed sensors

\begin{tabular}{ll}
\hline Sensor type & Data collected \\
\hline Motion sensor & Incidents of movements \\
Accelerometer & Mattress movements \\
Power sensor & Electrical power draw by appliances \\
Acoustic sensor & Water flows in pipes \\
Temperature sensor & Temperature readings \\
Humidity sensor & Humidity readings \\
Read switch & Doors open/closed \\
Pressure sensor & Sofa/couch being attended \\
\hline
\end{tabular}

\subsection{Intelligent Home Monitoring System}

Figure 1 illustrates the function of the smart home: data collection, analyses and presentation.

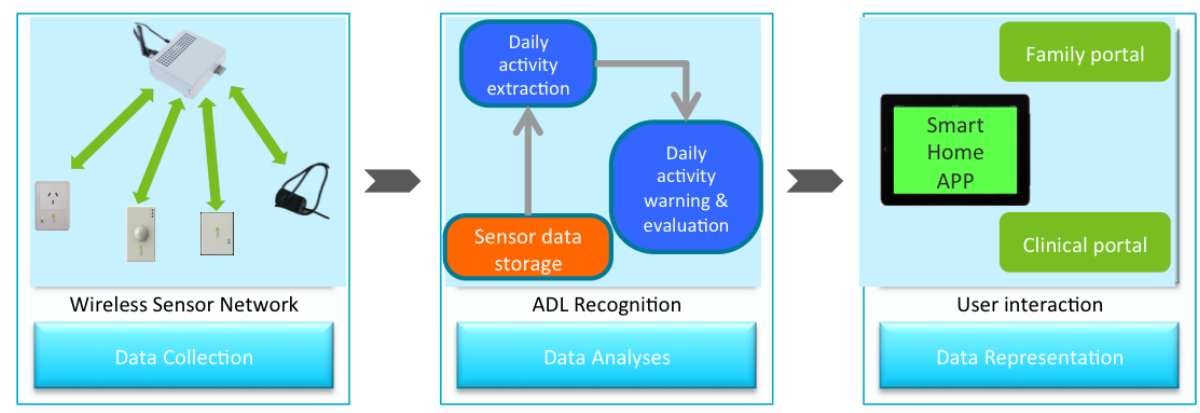

Fig. 1. Smart Home platform architecture

\section{Data Collection}

This module gathers continuously recorded, daily activity data of a person from the non-intrusive sensors deployed at invisible locations at home. Figure 2 presents the places where these sensors are likely to be located. 

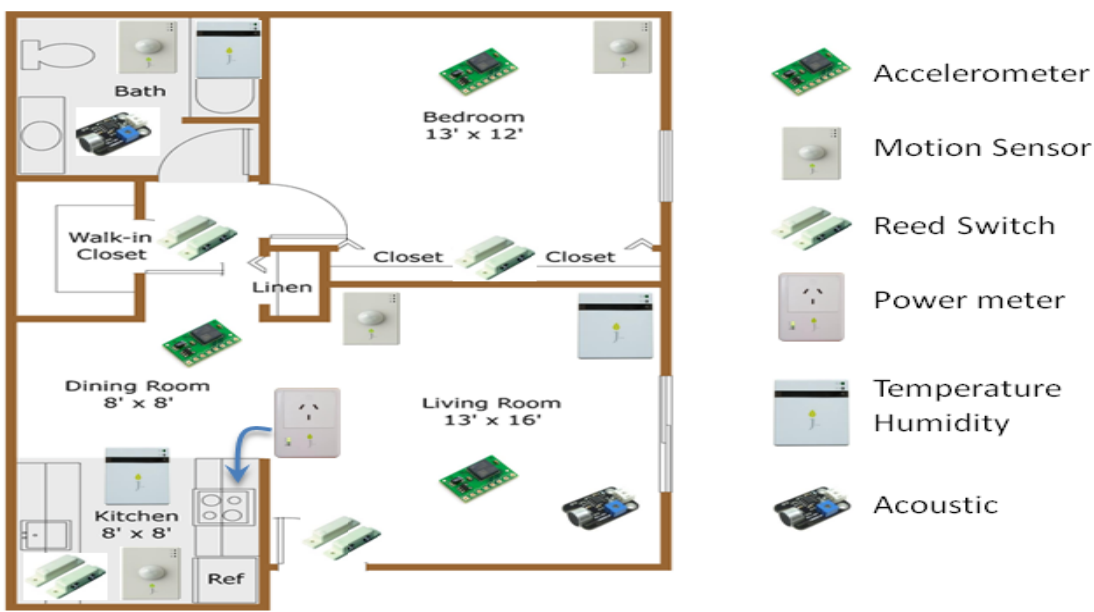

Fig. 2. Typical places where the sensors will be installed

\section{Data Analyses}

This module analyses raw sensor data captured to extract information about a person's routine behaviour and ADL. It will extract activity patterns and measure the person's physical, health and mental functions. Based on statistical data analysis and machine learning, it will provide warning/alert of abnormal situations in real time when such situation happens. By triangulating data from other sources, it is anticipated that the level and type of assistance that a person with early onset of dementia requires can be estimated and be provided to enable him or her to live independently as long as possible.

Raw sensor data is initially processed at the in-house local server. The output is converted to semantically meaningful representation of observed actions. The design and implementation of information presentation and service provision will benefit from semiotics, a well-established discipline of signs and human communication in social context. The semiotic framework will guide us in taking account of syntactic, semantic and pragmatic levels of system functions and service provision to the different stakeholders. In our system, sensors collect simple actions such as turn on/off the microwave, move in/out of the bathroom. These actions, along with their temporal features, are then transmitted to the database server for more complex activity extractions. An activity is a more complex motion pattern, typically composed of many actions and with longer durations. For example, turning on a microwave is considered an action, while preparing a meal is an activity. Typical activities of daily living, as a concept that is first established by Katz, include bathing, dressing, toileting, transferring, continence and feeding [5]. It is well known that maintaining ADL becomes a major focus for care of people with dementia. Table 2 shows a non-exhaustive list of ADLs that could be automatically extracted from the related sensors in our system. 
Table 2. Extracting ADLs from sensors

\begin{tabular}{|l|l|}
\hline \multicolumn{1}{|c|}{ Daily activities } & \multicolumn{1}{c|}{ Sensor types } \\
\hline Indoor walking/wandering & Motion sensors \\
\hline Sleep & Motion sensors, accelerometer \\
\hline Preparing meals & Motion, Power, Acoustic sensors \\
\hline Washing cloth & Power, Motion sensors, Reed switch \\
\hline Visiting bathroom & Motion, Temperature, Humidity sensors \\
\hline House cleaning & Motion, Power, Acoustic sensors \\
\hline Postural transfer & Pressure, Motion sensor \\
\hline
\end{tabular}

\section{Wellness Determination Process through Monitoring Daily Activities}

Fig. 2 shows the schematic representation of the determination of wellness of elderly in the proposed Smart Home. The recognition of basic ADLs and determination of wellness of the elderly are described in the following Equations.

$$
\mathrm{P}(\mathrm{t} \mid \mathrm{c})=\frac{N_{c t}+1}{\sum_{t^{\prime} \in V}\left(N_{c t^{\prime}}+1\right)}=\frac{N_{c t}+1}{\sum_{t^{\prime} \in V} N_{c t^{\prime}}+K^{\prime}}
$$

where $\mathrm{N}_{\mathrm{ct}}$ is the number of times a particular sensor ID occurs in activity ' $\mathrm{c}$ '. $\mathrm{V}$ is the set of sensor IDs. $\mathrm{K}^{\prime}=|\mathrm{V}|$ is the number of unique sensor IDs. The first function $\left(\beta_{1}\right)$ indicates about the non-usage or inactive duration of the appliances, and the second function $\left(\beta_{2}\right)$ is about the over-usage of specific household appliances. The following are the details:

$$
\beta_{1}=1-\frac{t}{T} ; \beta_{2}=1+\left(1-\frac{T_{\alpha}}{T_{n}}\right)
$$

\section{Data Presentation}

In order to improve awareness and facilitate self monitoring, data will be presented in tablet PC or mobile phone. This will enable family members to gain an insight into the capacity and condition of living of their beloved ones; assist health care providers to get a whole picture of the person's daily lives, routines and health status. The awareness and information will facilitate the timely provision of intervention through telehealth and telecare.

The data will be presented to the person for whom home monitoring service is provided, to family care givers and to clinical professionals providing health services to the person. It can be used by the person for the purpose of self-monitoring, by care givers as a means for remote monitoring and care assistance, and by clinicians to support their diagnostic and treatment decision through examining the changes/trends in ambient and activities of daily living, psychological, behavioral and vital signs, respectively, for older people with early dementia, which is particularly useful for those living at home alone. For the later, we will provide a tablet-based application for them to self-monitor their daily activities and conduct physiological assessments regularly. 


\section{Implementation Studies}

The Smart Home system will be developed and implemented in the relevant population to develop and test the system implementation methods. The purpose of the trial is to (1) develop and fine-tune the technology; (2) understand the facilitators and barriers for acceptance and usage of the system; (3) test acceptance, usage and impact of the system on people with mild dementia and their family carers; (4) develop the relevant training and support services to foster adoption and usage; and (5) develop the business model for the commercial roll out of the solution to large population. These will be achieved in three stages.

\section{Stage 1. Develop and Fine-Tune the Technology, and Develop Understanding about Facilitators and Barriers for System Adoption}

This trial will be conducted in small population with five to ten people who suffer from mild dementia. The selection of sample will take into account a balance of genda, living environment and life style. Both engineers and system analysts will be involved in the trial. In addition to the data collected by the sensors, human factors for system implementation will also be collected through interview and observation. These data are important in guiding the development of user-friendly interface that will be accepted by the people with dementia and their family carers. The indicators for human factors include the interface design that is acceptable by the end users, the older people's preference for navigating the system and the type of devices with optimal opportunity to be accepted and used. The functions and messages that are useful for the intended population will also be identified.

\section{Stage 2. Pilot Trial of the System in Small Population Group}

After the initial validation, the system will be further implemented in a small population group of 30 to 50 people with dementia to further test the functionality, user acceptance, usage and effectiveness of the system. Any issues identified will be timely fixed, with system improved. This test and improvement process will be continuously conducted and evolved until enough evidence has been gathered to prove that the system has achieved its objectives.

\section{Stage 3. Large Scope Implementation to Develop the Business Model for Rolling Out the System}

After the efficacy of the system is validated, the solution will be rolled out in the scope of a city or province to further test its efficacy for large population. The relevant business model for technology roll out will be developed. This include costing model for the solution, implementation strategies, end user support and roles and responsibilities of the relevant stakeholder groups, etc.

\section{Conclusions}

This paper briefly outlines a comprehensive Smart Home solution to assist people with mild dementia to live at home. It first presents the technical solution, then the empirical technology development, pilot testing and implementation strategies for 
rolling out the system to the population. Different from the solutions with wearable devices, the sensors of our Smart Home are placed at various locations in the residency of a person, which will bring in the advantage of no intrusion thus comfort of use. We plan to develop and introduce the system through a rigorous technology development and implementation strategy and practice. The human factors that either facilitate or hinder the adoption of the Smart Home solution will be fully noticed with solution developed. The conduct of the project will contribute to Smart Home technology development and knowledge about effective ICT solutions to assist people with memory loss. It will also contribute to addressing the biggest challenge of population ageing in the current world - dementia, taking advantage of current advancement of ICT [7,8,9].

Acknowledgements. This work was financially supported by the National Soft Science Planning (2011GXQ4K029), and all sensor data will be collected via National Population and Reproductive Health Science Data Center of National Scientific Data Sharing Platform for Population and Health.

\section{References}

1. Zouba, N., Boulay, B., Bremond, F., Thonnat, M.: Monitoring Activities of Daily Living (ADLs) of Elderly Based on 3D Key Human Postures. Cognitive Vision (2008)

2. Sangwan, R.S., Qiu, R.G., Jessen, D.: Using RFID tags for tracking patients, charts and medical equipment within an integrated health delivery network. In: IEEE Networking, Sensing and Control (2005)

3. Cañas, J.M., Marugan, S., Marrón-Romera, M., Garcia, J.C.: Visual Fall Detection for Intelligent Spaces. In: 6th IEEE International Symposium on Intelligent Signal Processing (2009)

4. Zhang, Q., Karunanithi, M., Rana, R., Liu, J.: Determination of Activities of Daily Living of independent living older people using environmentally placed sensors. In: 2013 35th Annual International Conference of the IEEE Engineering in Medicine and Biology Society (EMBC), pp. 7044-7047 (2013), doi:10.1109/EMBC.2013.6611180

5. Plan, T.H.: Katz Activities of Daily Living (ADL), pp. 1-8 (2008)

6. National Population and Reproductive Health Science Data Center on, http: / / www. poprk. org/

7. Yu, P.: Aged care IT in Australia - the past, present and future. eJournal of Health Informatics 7(2), e2 (2012)

8. Yu, P.: A multi-method approach to evaluate health information systems. Studies in Health Technology and Informatics 160(2), 1231-1235 (2010)

9. Su, Y., et al.: Consumer-centered eHealth: challenges and opportunities for China. Advanced Science Letters 7, 257-260 (2012) 